\title{
dTCApFs, a derivative of a novel human hormone peptide, induces apoptosis in cancer cells through a mechanism involving loss of Golgi function
}

\author{
JOEL OHANA $^{1}$, UZIEL SANDLER $^{1,2}$, GIDEON KASS $^{1}$, SALOMON M. STEMMER $^{3}$ and YORAM DEVARY ${ }^{1}$ \\ ${ }^{1}$ Immune System Key (ISK) Ltd., Jerusalem 9746009; ${ }^{2}$ Department of Bio-Informatics, Lev Academic Center (JCT), \\ Jerusalem 91160; ${ }^{3}$ Sackler Faculty of Medicine, Tel Aviv University, Tel Aviv 69978, Israel
}

Received June 30, 2017; Accepted September 27, 2017

DOI: $10.3892 / \mathrm{mco} .2017 .1453$

\begin{abstract}
TCApFs (Nerofe ${ }^{\mathrm{TM}}$ ) is a 14-amino acid derivative of a longer hormone peptide, tumor-cells apoptosis factor (TCApF), which enters the cells through the T1/ST2 receptor. In the present study, the mechanism of action (MOA) of dTCApFs as an anticancer agent was investigated. Experiments were performed in pancreatic cancer cell lines, and immunofluorescent staining demonstrated that dTCApFs is located in the Golgi apparatus of treated cells. It was also demonstrated in pancreatic, breast and ovarian cell lines that dTCApFs treatment led to Golgi structural changes, loss of Golgi function, and molecular effects associated with endoplasmic reticulum (ER) stress, such as increased levels of C/EBP homologous protein, binding immunoglobulin protein (BiP), phosphorylated inositol-requiring enzyme 1 (pIRE1), and increased phosphorylation of eukaryotic translation initiation factor $2 \alpha$, and to the generation of reactive oxygen species, which was attenuated by ER stress inhibitors. Moreover, in these cell lines, long-term exposure to dTCApFs led to downregulation of spliced X-box-binding protein 1, which is an ER stress repair mechanism gene, downregulation of the Golgi anti-apoptotic protein, and reduced cell viability. In vivo studies using murine xenograft models of human pancreatic cancer verified the cell culture findings by demonstrating structural changes in the ER/Golgi and increased levels of pIREland BiP in dTCApFs-treated mice vs. the controls. Finally, human tissue samples from a patient who received dTCApFs for 11 months in a clinical trial were analyzed, and an increase was observed in the number of cells expressing pIRE1 and BiP post-treatment. In conclusion, we herein report a novel MOA for an anticancer agent involving triggering of apoptosis through induction of opposite effects: ER stress and downregulation of the ER stress repair mechanism. These findings provide the framework for the clinical evaluation of dTCApFs.
\end{abstract}

Correspondence to: Dr Yoram Devary, Immune System Key (ISK) Ltd., 9/7 Rosenblatt Street, Jerusalem 9746009, Israel

E-mail: ydevary@immunesk.com

Key words: apoptosis, cancer, GolgiStop, endoplasmic reticulum stress, hormone peptide, T1/ST2

\section{Introduction}

Tumor-cells apoptosis factor (TCApF) is a novel human 84-amino acid hormone peptide naturally expressed in the thymus, colon and frontal lobe of the brain (1). TCApF was identified through screening of the human genome with bioinformatics tools, and was found to have anticancer activity in acute myeloid leukemia (AML), glioblastoma, breast, lung and prostate cancer cell lines (1). Studies investigating the mechanism through which TCApF exerts its anticancer activity in AML demonstrated that it binds to the T1/ST2 receptor, which is a member of the Toll/Interleukin-1 receptor superfamily expressed in macrophages, dendritic cells and mast cells, and was implicated in various diseases, including cancer, inflammatory and cardiovascular diseases $(2,3)$. The T1/ST2 receptor is a stable marker of T-helper 2 (Th2) cells, but not of polarized thymocytes, and is important in Th2-associated immune response. Differential mRNA processing within the ST2 gene generates three ST2 isoforms, namely a full transmembrane receptor (ST2L), a truncated form associated with the membrane (ST2V), and a secreted soluble form (sST2) (4). The activation of the T1/ST2 receptor has been shown to lead to apoptosis in proliferating cells (but not in non-proliferating cells) through activation of caspases 8 and B-cell lymphoma (Bcl)-2-mediated apoptotic pathways (1).

dTCApFs (Nerofe ${ }^{\mathrm{TM}}$, Immune System Key Ltd., Jerusalem, Israel) is a short, 14-amino acid derivative of TCApF that retains its anticancer activity. The objective of the present study was to investigate the mechanism of action (MOA) of dTCApFs, referring particularly to its potential activity in solid tumors, in order to provide the necessary framework for clinical studies evaluating its utility as a potential treatment for advanced/metastatic solid tumors.

\section{Materials and methods}

$d T C A p F s$. dTCApFs was synthesized by Novetide Ltd. (Haifa, Israel) at Asia Chemical Industries Ltd. (Ramat Hovav, Beer Sheva, Israel). The polypeptide was assembled on a solid support using protected amino acids; the peptide was then cleaved from the resin and purified using preparative high-performance liquid chromatography, followed by desalting and counter-ion exchange to generate dTCApFs acetate. 
Cell lines. Human cell lines including PANC-1 (pancreatic cancer), OV-90 (ovarian cancer) and MDA-MB-231 (breast adenocarcinoma) were obtained from American Type Culture Collection and used within 6 months. Cell lines were maintained in DMEM (Thermo Fisher Scientific, Waltham, MA, USA) with $10 \%$ fetal bovine serum (FBS; Biological Industries, Beit Haemek, Israel), $50 \mu \mathrm{g} / \mathrm{ml}$ gentamycin sulfate (Biological Industries) and $250 \mu \mathrm{g} / \mathrm{ml}$ amphotericin B (Biological Industries).

Cell culture and dTCApFs treatment. For immunofluorescence (IF) assays, 10,000 PANC-1/MDA-MB-231/OV-90 cells were seeded into 8-well chamber slides (Nunc ${ }^{\circledR}$, Roskilde, Denmark) in $0.5 \mathrm{ml} \mathrm{10 \%} \mathrm{FBS} \mathrm{in} \mathrm{DMEM.} \mathrm{Following} \mathrm{incuba-}$ tion for $24 \mathrm{~h}$, the medium was changed to $2.5 \%$ FBS in DMEM and $5 \%$ mannitol, and the cells were treated with $25 \mu \mathrm{g} / \mathrm{ml}$ dTCApFs for $48 \mathrm{~h}$, after which time another dose of dTCApFs $(25 \mu \mathrm{g} / \mathrm{ml})$ was added to the medium. After an additional $24 \mathrm{~h}$, cells were collected for IF analysis.

IF.For IF studies, cells were grown on glass chamber slides, fixed with 3\% formaldehyde in phosphate-buffered saline (PBS), permeabilized with $0.25 \%$ Triton X-100 in PBS, blocked with $1 \%$ bovine serum albumin in PBS plus Tween-20, incubated overnight at $4^{\circ} \mathrm{C}$ with the primary antibody in blocking media, and stained for $1 \mathrm{~h}$ with secondary antibody. The primary antibodies used included anti-dTCApFs (1:1,000 rabbit, polyclonal, custom made by Genemed Synthesis Inc., San Antonio, TX, USA) and anti-soluble T1/ST2 (1:500 rabbit, polyclonal, custom made by Genemed Synthesis Inc.). Additional primary antibodies used included anti-58k Golgi protein antibody (ab27043, 1:500 mouse monoclonal, Abcam Cambridge, UK), anti- $\gamma$-adaptin (ab11328, 1:500 mouse monoclonal, Abcam), anti-coatomer subunit $\beta$ ( $\beta$-COP) antibody (ab6323, 1:250 mouse monoclonal, Abcam), anti-inositol-requiring enzyme (IRE)1 antibody (9F2, ab96481, 1:500 mouse monoclonal, Abcam), antibody against Golgi anti-apoptotic protein (GAAP) (ab169461, 1:100 mouse polyclonal, Abcam), and anti-C/EBP homologous protein (CHOP) antibody (no. 2895), anti-binding immunoglobulin protein $(\mathrm{BiP})(\mathrm{C} 50 \mathrm{~B} 12)$ rabbit mAB (no. 3177, 1:400 rabbit monoclonal, Cell Signaling Technology, Danvers, MA, USA), and anti-phospho-eukaryotic translation initiation factor (eIF) $2 \alpha$ (Ser51) (D9G8) XP rabbit mAB (no. 3398, pEIF2a rabbit monoclonal, Cell Signaling Technology). Secondary antibodies included anti-rabbit IgG $(\mathrm{H}+\mathrm{L}), \mathrm{F}\left(\mathrm{ab} \mathrm{b}^{\prime}\right) 2$ Fragment (no. 4413, 1:1,000 goat, Cell Signaling Technology), and anti-mouse IgG (H+L), F(ab')2 fragment (no. 4408, 1:1,000 goat, Cell Signaling Technology), goat anti-rabbit IgG $\mathrm{H}+\mathrm{L}$ (ab150077) and goat anti-mouse (ab150118) (both diluted 1:1,000, Abcam). Slides were mounted with fluoroshield mounting medium with 4',6-diamidino-2-phenylindole (DAPI; ab104139, Abcam). The microscopes used included BX41 and FV-1200 (Olympus, Tokyo, Japan).

Immunohistochemistry (IHC). IHC examination of paraffin-embedded tumor sections was performed by deparaffinizing the sections with washes of xylene, and rehydrating them with serial washes of absolute ethanol, $95 \%$ ethanol, and then with distilled water. Antigen retrieval was performed by boiling the sections in Tris-EDTA ( $\mathrm{pH}$ 9) for ST2 staining and in acetate $(\mathrm{pH} 6)$ for other antibodies. Endogenous peroxidase was blocked with $3 \%$ oxygen peroxidase. Non-specific binding was blocked with Background Buster (Innovex Biosciences, Richmond, CA). The primary antibody was incubated on slide overnight at $4^{\circ} \mathrm{C}$ in SignalStain antibody diluent (Cell Signaling Technology). Secondary antibody binding and 3,3'-diaminobenzidine (DAB) development were performed the following day. The nuclei were stained with hematoxylin. The slides were dehydrated, mounted and visualized under an Olympus BX41 microscope (Olympus) with a CCD camera. Primary antibodies included those used in the IF experiments, as well as an antibody against the full-length ST2 (prepared for the present study by Genemed Synthesis Inc.). Secondary antibodies included anti-rabbit horseradish peroxidase (HRP; Cell Signaling Technology) diluted in SignalStain ${ }^{\circledR}$ Boost IHC Detection Reagent (Cell Signaling Technology) with DAB as the substrate, and Eukitt ${ }^{\circledR}$ as the mounting media (both from Sigma-Aldrich, St. Louis, MO, USA).

Reverse transcription-polymerase chain reaction (RT-PCR) analysis, ELISA, dihydroethidium (DHE) assay, western blotting and cell viability studies. RT-PCR analysis was performed as previously described (1). For the ELISA studies, 100,000 cells were incubated in 6-well plates in $2 \mathrm{ml}$ of $10 \%$ FBS in DMEM medium and, $24 \mathrm{~h}$ later, the medium was changed to $1 \mathrm{ml}$ of $2.5 \% \mathrm{FBS}$ and $5 \%$ mannitol in DMEM. Cells were treated with dTCApFs as in the IF experiments, and the supernatant was collected. Cell debris was removed by centrifugation. Soluble T1/ST2 peptide (for calibration) or cell supernatant was added to 96-well microplate (MaxiSorp; Nunc) and incubated in PBS at $4^{\circ} \mathrm{C}$ overnight. The primary antibody was anti-soluble T1/ST2 (as for the IF experiments), and the secondary antibody was anti-rabbit IgG HRP-linked (Cell Signaling Technology). For DHE experiments, cells were seeded at concentrations of 20,000/well into black 96-well plates in $100 \mu \mathrm{l}$ of growing media (DMEM with $4,500 \mathrm{mg} / \mathrm{l}$ glucose, $4 \mathrm{mM}$ L-glutamine, $1 \mathrm{mM}$ sodium pyruvate, $10 \%$ FBS, $250 \mathrm{ng} / \mathrm{ml}$ amphotericin $\mathrm{B}, 100 \mathrm{U} / \mathrm{ml}$ penicillin, and $100 \mu \mathrm{g} / \mathrm{ml}$ streptomycin) overnight. The medium was then changed to the growing media with or without dTCApFs (50 $\mu \mathrm{g} / \mathrm{ml})$, and with or without 4-phenylbutyrate (PBA; $5 \mu \mathrm{M})$. Following overnight incubation, the cells were incubated with growing media containing $10 \mu \mathrm{M}$ DHE, incubated for $20 \mathrm{~min}$ and washed 3 times with PBS. The plates were read using a fluorescent spectrophotometer (BIO-TEK Synergy HT, BioTek Instruments, Inc., Winooski, VT, USA) with an excitation/emission wavelength of $590 / 530 \mathrm{~nm}$. The experiment was performed in triplicate. For western blot analysis, the cells were grown as described above. After treatment [dCTApFs, PBA, control (5\% mannitol)], the cells were washed twice with PBS and collected (10 min, $300 \mathrm{x} \mathrm{g}$, $4^{\circ} \mathrm{C}$ ). Lysis buffer containing RIPA buffer and phosphatase inhibitors (both from Sigma-Aldrich), and protease inhibitor (Sigma-Aldrich), were added for $10 \mathrm{~min}$ and the cells were incubated for $10 \mathrm{~min}$ on ice. The cells were then broken using a $21 \mathrm{G}$ needle. Supernatant was collected after centrifuging the cells $(15,000 \mathrm{x} \mathrm{g})$ for $10 \mathrm{~min}$, LDS sample buffer and reducing agent (both from Invitrogen, Carlsbad, CA, USA) were added to each sample. The samples were boiled 


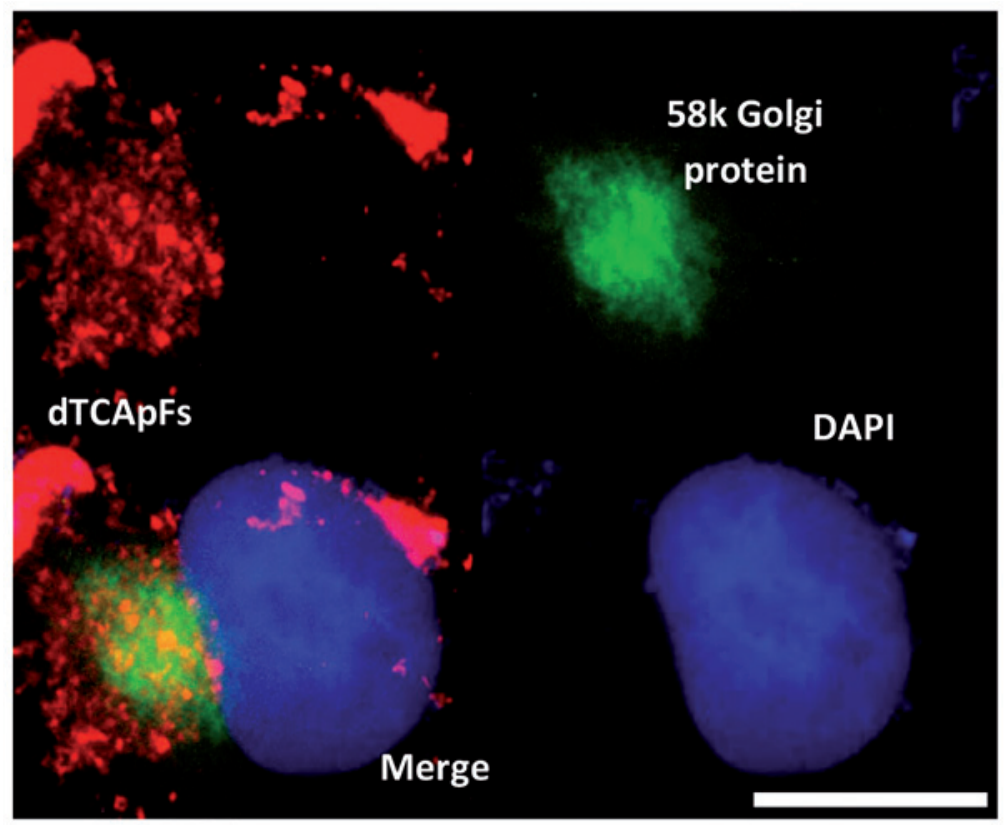

Figure 1. dTCApFs is located in the Golgi apparatus of treated cells. In PANC-1 cells treated with dTCApFs $(25 \mu \mathrm{g} / \mathrm{ml})$ for $72 \mathrm{~h}$, IF staining showed that dTCApFs was localized in/next to the Golgi apparatus. dTCApFs, red; $58 \mathrm{kDa}$ golgi protein, green; DAPI (DNA), blue. Scale bar, $25 \mu \mathrm{m}$. dTCApFs, 14 -amino acid derivative of tumor-cells apoptosis factor; IF, immunofluorescence; DAPI, 4',6-diamidino-2-phenylindole.
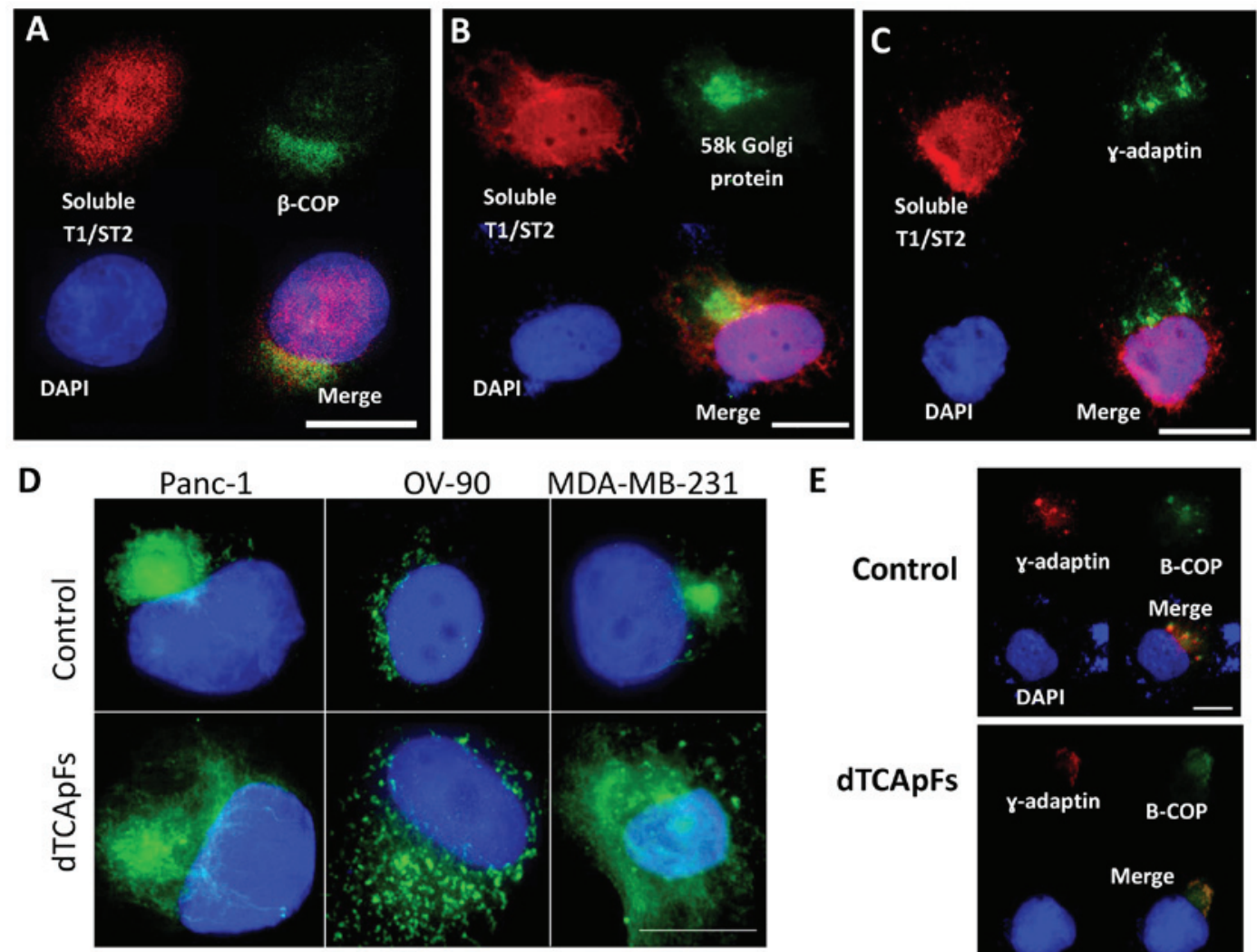

E

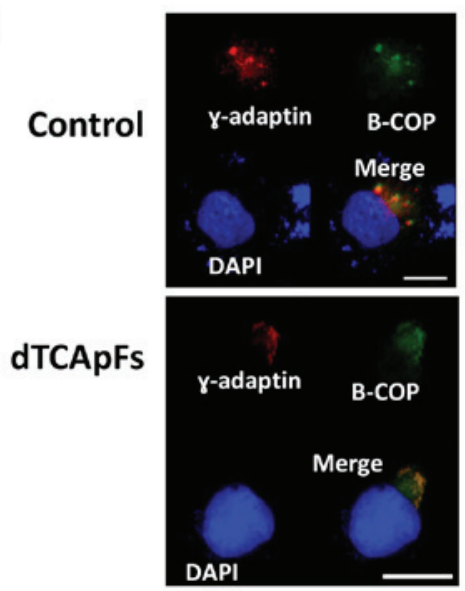

Figure 2. dTCApFs causes a structural change in the Golgi apparatus, in untreated cells, soluble T1/ST2 is in the ER/Golgi, whereas in dTCApFs-treated cells it is in the nucleus, and the Golgi apparatus is diffused and separated from the ER. (A) Soluble T1/ST2 was detected in the ER of untreated PANC-1 cells (IF staining) Soluble T1/ST2, red; DAPI (DNA), blue, $\beta$-COP (ER marker), green. (B) Soluble T1/ST2 was detected in the Golgi apparatus of untreated PANC-1 cells (IF staining). Soluble T1/ST2, red; DAPI (DNA), blue; $58 \mathrm{kDa}$ Golgi protein, green. (C) Soluble T1/ST2 was detected (IF staining) only in the nucleus of PANC-1 cells treated with dTCApFs $(25 \mu \mathrm{g} / \mathrm{ml}$ for $48 \mathrm{~h}$ followed by an additional dose of $25 \mu \mathrm{g} / \mathrm{ml}$ for a further $24 \mathrm{~h}$ ). Soluble T1/ST2, red; DAPI (DNA), blue; $\gamma$-adaptin (a Golgi marker), green. (D) In MDA-MB-231 cells treated with dTCApFs ( 25 or $50 \mu \mathrm{g} / \mathrm{ml}$ for $24 \mathrm{~h}$ followed by an additional dose of $25 \mathrm{or} 50 \mu \mathrm{g} / \mathrm{ml}$ for a further $72 \mathrm{~h}$ ), the Golgi apparatus structure was diffused, compared with controls (no dTCApFs treatment). DAPI (DNA), blue; 58 kDa Golgi protein, green. (E) In PANC-1 cells treated with dTCApFs $(25 \mu \mathrm{g} / \mathrm{ml}$ for $48 \mathrm{~h}$ followed by an additional dose of $25 \mu \mathrm{g} / \mathrm{ml}$ for a further $24 \mathrm{~h})$, the Golgi apparatus structure was separated from the ER. DAPI (DNA), blue; $\gamma$-adaptin (a Golgi marker), red; $\beta$-COP (ER marker), green. Scale bars in all the panels, $25 \mu \mathrm{m}$. dTCApFs, 14-amino acid derivative of tumor-cells apoptosis factor; ER, endoplasmic reticulum; DAPI, 4',6-diamidino-2-phenylindole; $\beta$-COP, coatomer subunit $\beta$; IF, immunofluorescence. 
A
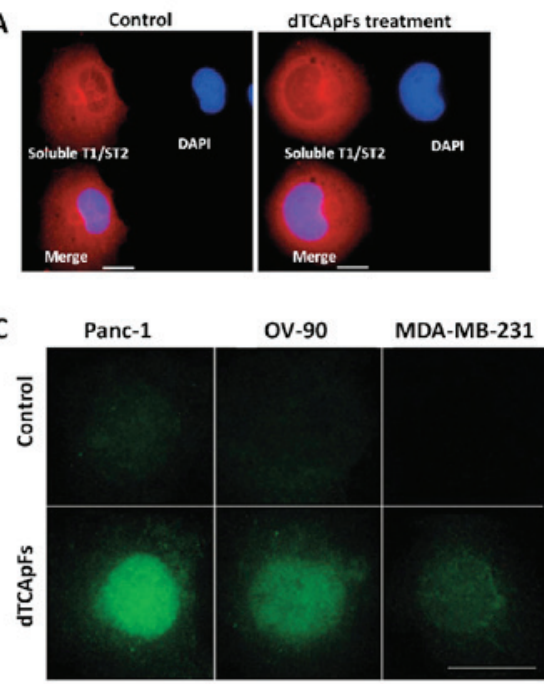

E

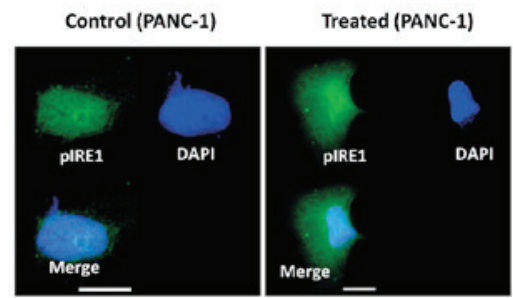

G

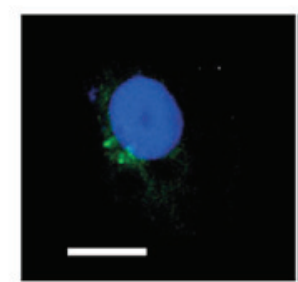

dTCApF PBA:
dTCApFs treatment

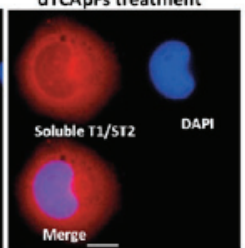

B

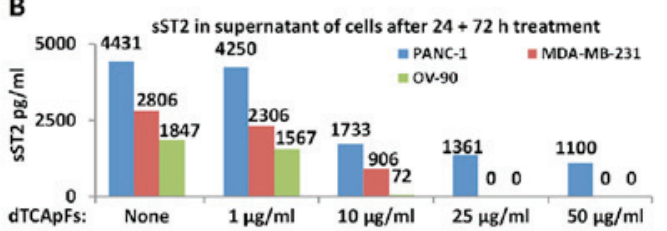

D

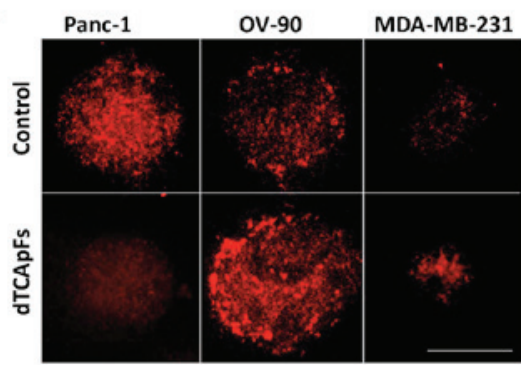

F
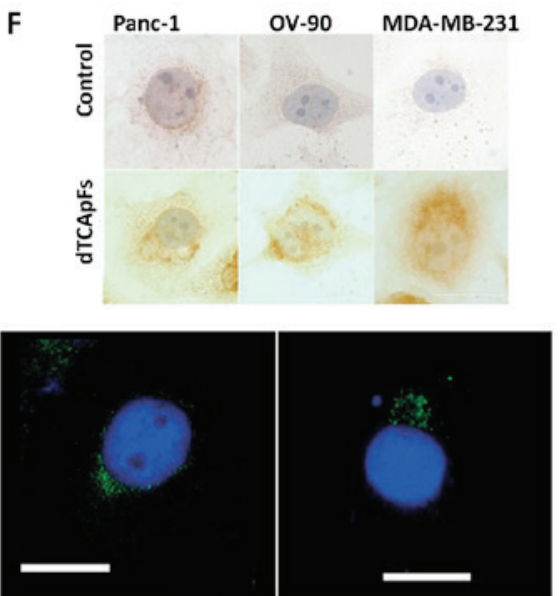

$+$

H

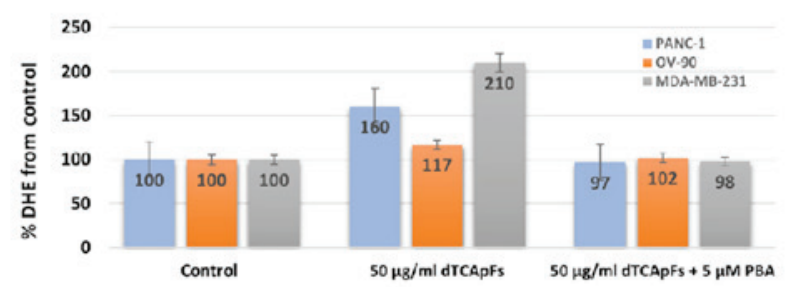

I

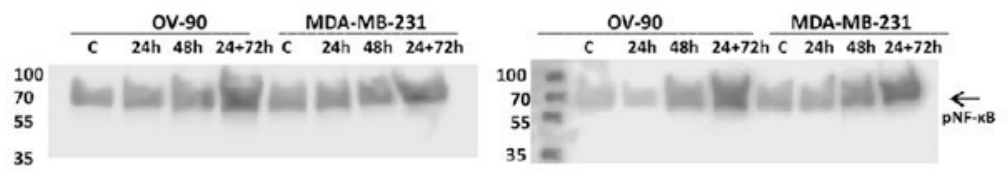

Figure 3. The dTCApFs-induced structural change in the Golgi apparatus results in loss of Golgi function and ER stress, In treated cells, the cellular levels of proteins (soluble T1/ST2) remain the same, whereas their secretion from the cells decreases, and molecular effects associated with ER stress are observed. (A) The soluble T1/ST2 levels were similar between untreated and treated (dTCApFs $50 \mu \mathrm{g} / \mathrm{ml}$ for $24 \mathrm{~h}$ ) PANC-1 cells (IF staining). Soluble T1/ST2; red; DAPI (DNA), blue. Scale bar, $25 \mu \mathrm{m}$. (B) Soluble T1/ST2 levels in the supernatant of cells treated with dTCApFs for $24 \mathrm{~h}$ followed by another dose for $72 \mathrm{~h}$, decreased with increasing concentrations of dTCApFs (ELISA). (C-E) IF/IHC staining of untreated and treated cells. For PANC-1 cells, treatment involved incubation with dTCApFs $50 \mu \mathrm{g} / \mathrm{ml}$ for $48 \mathrm{~h}$, followed by an additional dose of $50 \mu \mathrm{g} / \mathrm{ml}$ for $24 \mathrm{~h}$. For the OV-90 and MDA-MB-231 cell lines, the dTCApFs concentration was $25 \mu \mathrm{g} / \mathrm{ml}$. The cells exhibited increased levels of (C) CHOP, (D) BiP and (E) pIRE1 in the cytoplasm and (F) e1F2a phosphorylation with dTCApFs treatment. (G) The Golgi apparatus became diffused in OV-90 cells treated with dTCApFs (50 $\mu \mathrm{g} / \mathrm{ml}$ for $24 \mathrm{~h}$ followed by an additional dose of $50 \mu \mathrm{g} / \mathrm{ml}$ for $72 \mathrm{~h}$ ) compared with controls. Treatment with PBA ( $5 \mu \mathrm{M}$ for $48 \mathrm{~h}$ ) did not affect the Golgi apparatus, and treatment with both dTCApFs and PBA (in the concentrations described above) attenuated the effect of dTCApFs. Scale bars, $25 \mu \mathrm{m}$. (H) PANC-1, OV-90 and MDA-MB-231 cells were treated with dTCApFs (50 $\mu \mathrm{g} / \mathrm{ml})$ overnight, after which ROS levels were determined using the DHE assay and compared to untreated cells (controls). Treatment with dTCApFs $(50 \mu \mathrm{g} / \mathrm{ml}) \mathrm{plus} 5 \mu \mathrm{M} \mathrm{PBA}$ attenuated the observed ROS increase. Experiments were performed in triplicate. Error bars represent standard deviation (SD). (I) NF-kB and pNF-kB levels increased with dTCApFs treatment. OV-90, and the MDA-MB-231 cells were treated with dTCApFs $(50 \mu \mathrm{g} / \mathrm{ml})$ for the times indicated (for control, cells were incubated with 5\% mannitol for $24 \mathrm{~h}$ ) and cell lysates were subjected to western blot analysis. dTCApFs, 14-amino acid derivative of tumor-cells apoptosis factor; CHOP, C/EBP homologous protein; BiP, binding immunoglobulin protein; pIRE1, phosphorylated inositol-requiring enzyme 1; eIF2 $\alpha$, eukaryotic translation initiation factor $2 \alpha$; PBA, 4-phenylbutyrate; DHE, dihydroethidium; ER, endoplasmic reticulum; DAPI, 4',6-diamidino-2-phenylindole; $\beta$-COP, coatomer subunit $\beta$; IF, immunofluorescence; IHC, immunohistochemistry; ROS, reactive oxygen species; NF- $\mathrm{kB}$, nuclear factor- $\mathrm{\kappa}$. 
A

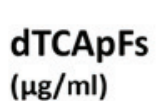

$(\mu \mathrm{g} / \mathrm{ml})$

sXBP1 $\rightarrow$
OV-90

C $\quad \begin{array}{llllllllll}10 & 25 & 50 & 75 & \text { C } & 5 & 10 & 25 & 50 & 75\end{array}$
B

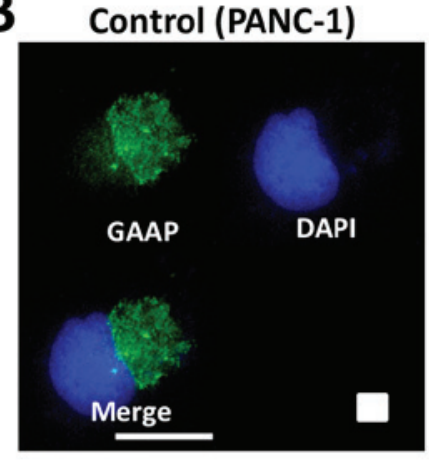

Treated (PANC-1)

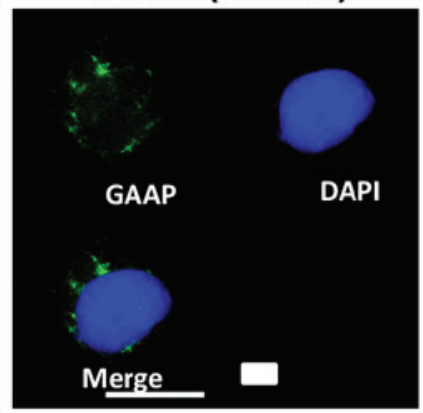

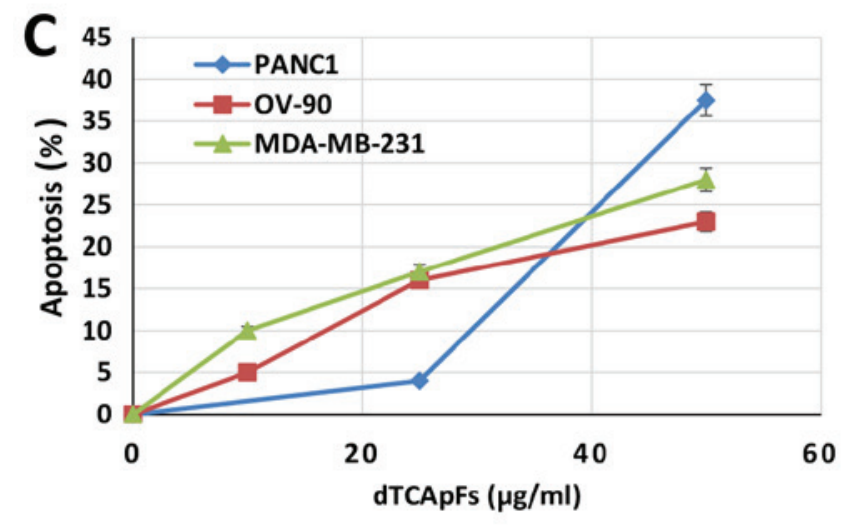

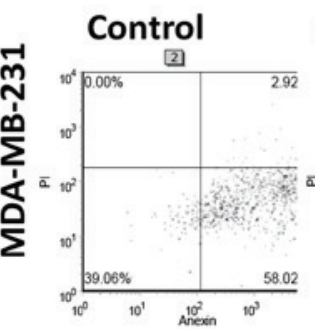

dTCApFs $(50 \mu \mathrm{g} / \mathrm{ml})$
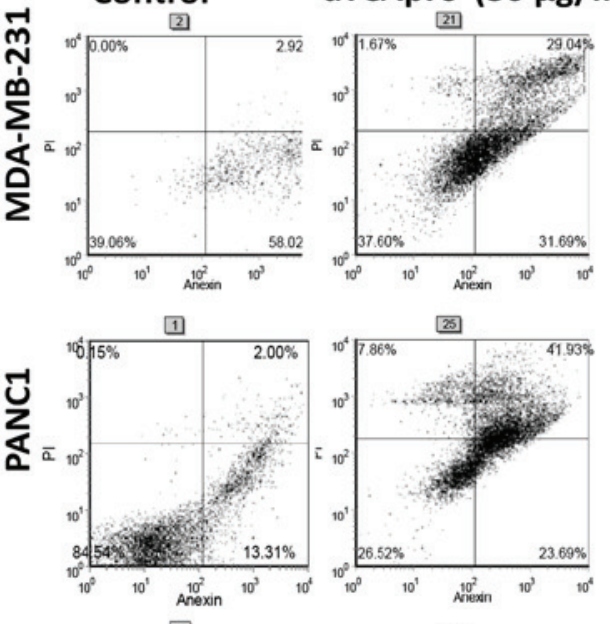

16
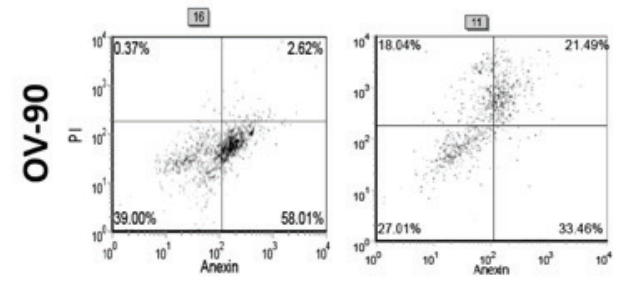

Figure 4. Long-term exposure to dTCApFs downregulates the ER stress repair mechanism and as a result apoptosis is induced. In treated cells, the sXBP1 and GAAP levels decreased. Cell viability also decreased. (A) RT-PCR-detected sXBP1 levels in OV-90, PANC-1 and MDA-MB-23 cells treated with dTCApFs for $72 \mathrm{~h}$ decreased with increasing dTCApFs concentrations (equal amounts of RNA/cDNA were used in the analysis). (B) The GAAP levels were higher in dTCApFs-treated PANC-1 cells ( $25 \mu \mathrm{g} / \mathrm{ml}$ for $48 \mathrm{~h}$ followed by an additional dose of $25 \mu \mathrm{g} / \mathrm{ml}$ for a further $24 \mathrm{~h}$ ) compared with untreated cells (IF staining), Scale bar, $25 \mu \mathrm{m}$. (C) Apoptosis was observed in PANC-1, OV-90 and MDA-MB-231 cells treated with increasing concentrations of dTCApFs for 24 h followed by another dTCApFs dose for $72 \mathrm{~h}$. Viability was measured with the Annexin V FITC assay. Error bars, standard deviation. dTCApFs, 14-amino acid derivative of tumor-cells apoptosis factor; ER, endoplasmic reticulum; RT-PCR, reverse transcription-polymerase chain reaction; sXBP1, spliced X-box-binding protein 1; GAAP, Golgi anti-apoptotic protein; IF, immunofluorescence.

for $5 \mathrm{~min}$ and loaded to 4-20\% Tris-Glycine gel (Nusep, New South Wales, Australia). Western blot analysis was performed according to standard procedures. The primary antibodies used included anti-nuclear factor (NF)- $\mathrm{B}$ and anti-pNF- $\kappa$ B (SC-3033 and SC-8242, respectively, Santa Cruz Biotechnology, Dallas, TX, USA). The secondary antibody was anti-rabbit IgG HRP-linked (Cell Signaling Technology).

For viability assays, cells (PANC-1/MDA-MB-231/OV-90) were seeded at concentrations of 80,000 cells/flask into $75 \mathrm{~cm}^{2}$ in $25 \mathrm{ml} \mathrm{10 \%} \mathrm{FBS} \mathrm{in} \mathrm{DMEM,} \mathrm{and} 24 \mathrm{~h}$ later the medium was changed to $25 \mathrm{ml} 2.5 \%$ FBS in DMEM with dTCApFs (0, 25, 50 or $75 \mu \mathrm{g} / \mathrm{ml}$ ) in $5 \%$ mannitol. After $48 \mathrm{~h}$, another dose of dTCApFs (at the same concentration as the initial dose) was added to each well and the cells were incubated with dTCApFs for another $24 \mathrm{~h}$. Viability was measured using the Annexin
V FITC assay (Medical \&Biological Laboratories Co. Ltd., Nagoya, Japan (according to the manufacturer's instructions, and was read using flow cytometry.

Murine xenograft models of human pancreatic cancer. A total of $8 \mathrm{Hsd}$ :Athymic Nude-Foxn1nu female mice (age, 5 weeks; weight, $20 \mathrm{gr}$ ), acquired from Harlan Laboratories Israel Ltd. (Jerusalem, Israel), were injected subcutaneously with $8 \times 10^{6}$ PANC-1 cells. dTCApFs treatment commenced after the tumor size had reached $0.5 \mathrm{~cm}$ at the largest dimension. The mice received intraperitoneal injections of dTCApFs $300 \mu \mathrm{g}$ per 20 gr 3 times a week. During treatment, tumor size was documented. At the end of treatment, the mice were euthanized by isofluran exposure (Primal Critical Care, Bethlehem, PA, USA) followed by cervical dislocation. Serum was collected for ELISA and the tumors were placed in $4 \%$ formaldehyde 
A

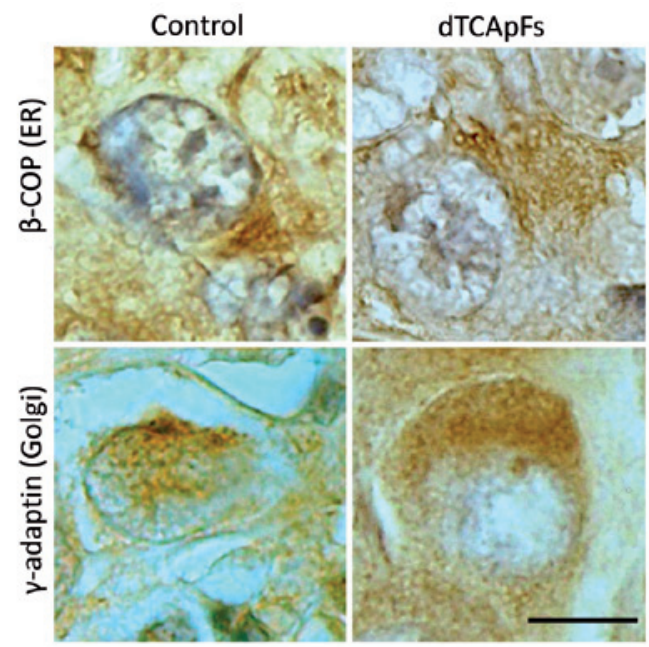

B

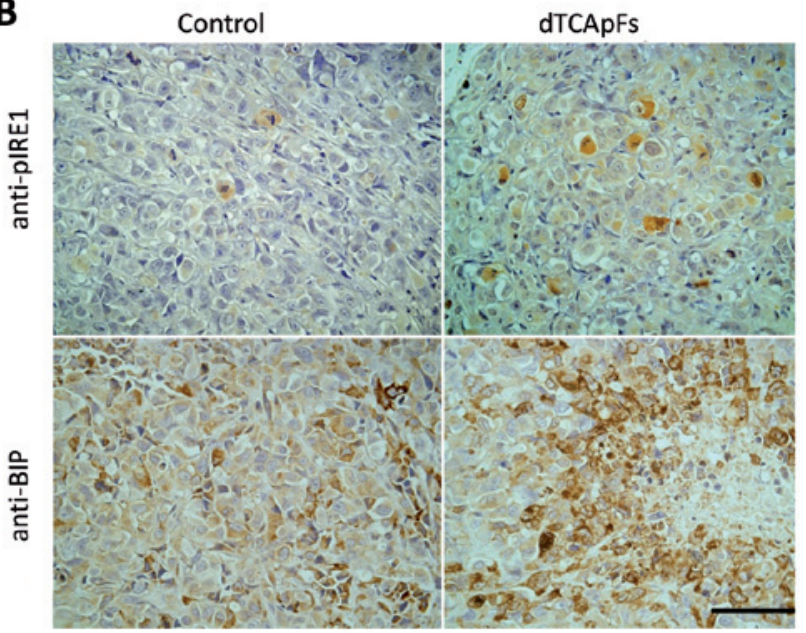

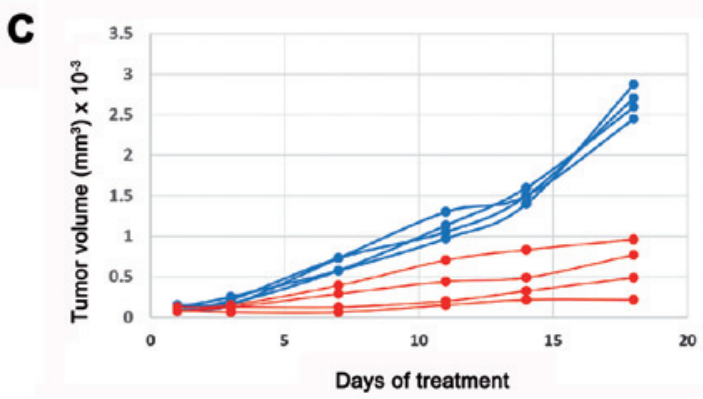

Figure 5. PANC-1 xenograft murine model studies showing that dTCApFs causes a structural change in the ER/Golgi, ER stress, and decreased tumor size. (A) IHC staining using anti- $\beta$ COP antibody (ER marker) or anti- $\gamma$-adaptin (Golgi marker) of tumor sections from untreated and dTCApFs-treated PANC-1 xenograft mice model demonstrating diffused ER/Golgi with dTCApFs treatment. Scale bar, $12 \mu \mathrm{m}$. (B) IHC staining with anti-pIER1 or anti-BiP of tumor sections from untreated and dTCApFs-treated PANC-1 murine xenograft model demonstrating increase in the number of cells expressing these ER stress markers. Scale bar, $50 \mu \mathrm{m}$. (C) Tumor size over time in PANC-1 mouse xenograft model that were dTCApFs-treated (15 mg/kg intraperitoneally twice a week) vs. controls (treatment with 5\% mannitol). Eight nude mice were inoculated subcutaneously with 1 million PANC-1 cells. When the tumor size exceeded 64 $\mathrm{mm}^{3}$, the animals were randomly assigned to treatment vs. control and tumor size was measured over time. Each line represents one animal in the experiment. dTCApFs, 14-amino acid derivative of tumor-cells apoptosis factor; ER, endoplasmic reticulum; IHC, immunohistochemistry; $\beta$-COP, coatomer subunit $\beta$; $\mathrm{BiP}$, binding immunoglobulin protein; pIRE1, phosphorylated inositol-requiring enzyme 1.

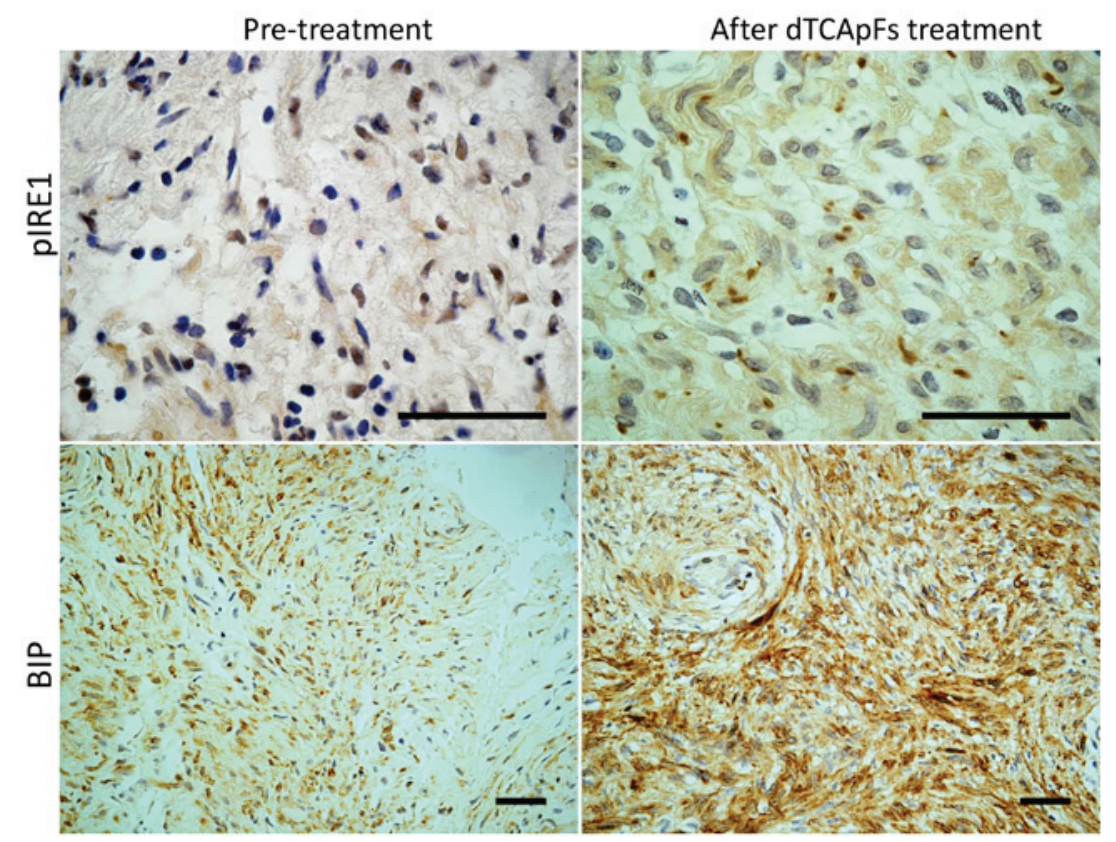

Figure 6. dTCApFs-induced ER stress in tumor samples from a patient treated with dTCApFs. IHC-stained samples pre- and post-treatment from a patient with spinal cord neoplasm who received dTCApFs 3 times a week at increasing concentrations of 12,24 , and $48 \mathrm{mg} / \mathrm{m}^{2}$ for 11 months showing an increase in the number of cells expressing pIRE1 and BiP. Scale bars, $50 \mu \mathrm{m}$. dTCApFs, 14-amino acid derivative of tumor-cells apoptosis factor; ER, endoplasmic reticulum; IHC, immunohistochemistry; BiP, binding immunoglobulin protein; pIRE1, phosphorylated inositol-requiring enzyme 1. 

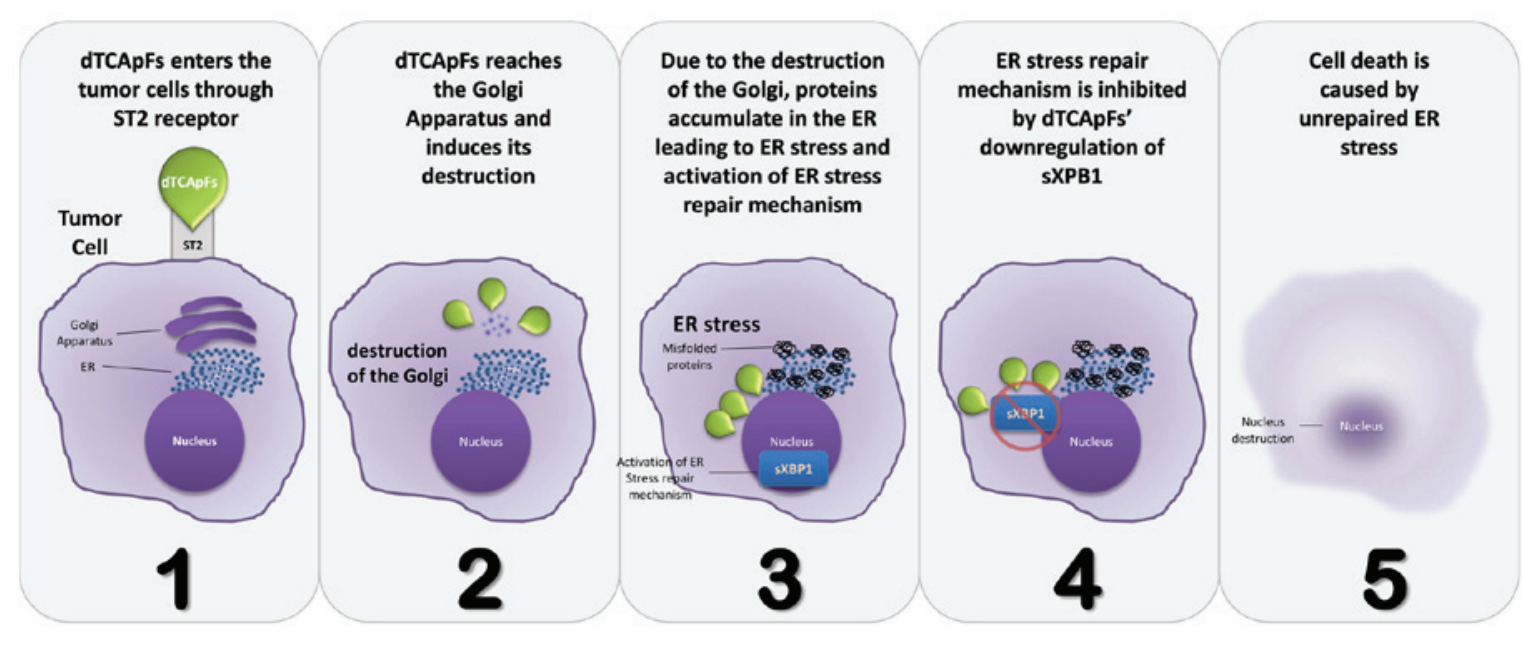

Figure 7. Schematic model of the mechanism of action of dTCApFs. dTCApFs, 14-amino acid derivative of tumor-cells apoptosis factor; ER, endoplasmic reticulum; sXBP1, spliced X-box-binding protein 1.

and sent to Pathovet Ltd. (Rehovot, Israel), where slides were prepared for IHC analysis. To determine the effect of dTCApFs on tumor size, the mice were injected subcutaneously with $1 \times 10^{6}$ PANC-1 cells. After the tumor volume had exceeded 64 $\mathrm{mm}^{3}$, the mice were randomly divided into dTCApFs treatment $(\mathrm{n}=4,15 \mathrm{mg} / \mathrm{kg}$ dTCApFs administered intraperitoneally) and control $(n=4,5 \%$ mannitol) groups, and the tumor size was measured every 4 days.

All animal studies were approved by and performed in accordance with the guidelines of the Institutional Animal Care and Use Committee.

Tumor tissue from a dTCApFs-treated patient. Samples before and after dTCApFs treatment were obtained from a patient who participated in a phase I study investigating dTCApFs in patients with pathologically confirmed locally advanced and/or metastatic solid tumors, who failed/could not tolerate standard therapy (personal communication). For this patient, a pre-treatment tumor sample was obtained from a biopsy of the primary tumor in the spinal cord and a post-treatment tumor sample was obtained following a partial sacrectomy. The phase I study in which this patient participated (clinicaltrials.gov identifier: NCT01690741) was approved by the Institutional Review Board of Rabin Medical Center and the Ministry of Health of Israel, it was conducted in accordance with the Declaration of Helsinki, and all the patients had signed an informed consent prior to enrollment.

\section{Results}

Cell culture studies. In order to elucidate the MOA of the anticancer activity of dTCApFs, we first investigated (by IF staining) its localization in PANC-1 cells. dTCApFs was found to be located in the Golgi apparatus of treated cells (Fig. 1).

IF staining was then used to investigate the subcellular changes induced by dTCApFs; the location of its soluble receptor (soluble T1/ST2) and the structure of the Golgi apparatus in treated vs. untreated cells were examined. Prior to treatment, soluble T1/ST2 was found in the nucleus, the ER, and the Golgi apparatus (Fig. 2A and B). Following dTCApFs treatment, soluble T1/ST2 was found only in the nucleus (Fig. 2C). Confocal microscopy studies using the $58-\mathrm{kDa}$ Golgi protein and DAPI for DNA staining, demonstrated that, in MDA-MB-23 cells, the Golgi apparatus was intact without dTCApFs treatment and diffused following dTCApFs treatment (Fig. 2D). Similar results were observed in the OV-90 and PANC-1 cell lines (data not shown). Using DAPI, the Golgi marker $\gamma$-adaptin and the ER marker $\beta$-COP in PANC-1 cells, it was demonstrated that the Golgi apparatus structure became separated from the ER following dTCApFs treatment (Fig. 2E).

Subsequently, it was investigated whether the structural changes observed in the Golgi apparatus were associated with loss of Golgi function and ER stress. To this end, IF staining was used to determine the levels of soluble T1/ST2 in untreated and dTCApFs-treated PANC-1cells, and ELISA experiments to determine the levels of soluble T1/ST2 in the supernatant of untreated and treated cells (PANC-1, OV-90 and MDA-MB-23) (Fig. 3A and B). With dTCApFs treatment, the levels of soluble T1/ST2 inside the cells remained the same (Fig. 3A), whereas its secretion from the cells into the supernatant decreased (Fig. 3B), suggesting loss of Golgi function. To further investigate the possibility of ER stress-related MOA, IF/IHC was used to evaluate the known molecular effects of such stress, including increased levels of CHOP [a transcription factor in the ER stress response (5)] and BiP [an abundant ER chaperone (6)] in the cells, expression of pIRE1 [an ER stress sensor protein (7)] in the cytoplasm and increased phosphorylation of eIF2a [which represses protein synthesis during ER stress response (8)]. As shown in Fig. 3C-F, dTCApFs treatment was associated with all these effects. Using IF staining in OV-90 cells revealed that the structural changes in the Golgi apparatus observed in dTCApFs-treated cells (but not in untreated controls), were not observed when the cells were incubated with dTCApFs and the ER stress inhibitor PBA (Fig. 3G). Similar results were observed with the OV-90 cell line (data not shown). The generation of reactive oxygen species (ROS), which has been associated with ER stress, was also evaluated in PANC-1, OV-90, and MDA-MB-23 cells treated with dCTApFs. An increase was found in ROS levels 
(by 1.2-2.1-fold) with dCTApFs treatment, which was attenuated with PBA (Fig. 3H), further implicating ER stress in the dCTApFs MOA. In addition, western blotting was used to evaluate the effect of dCTApFs treatment on the levels of NF- $\mathrm{BB}$ and $\mathrm{pNF}-\kappa \mathrm{B}$, both of which are implicated in ER stress. The experiment was performed on the OV-90 and MDA-MB-231 cell lines, and both cell lines were found to exhibit increased $\mathrm{NF}-\kappa \mathrm{B}$ and $\mathrm{pNF}-\kappa \mathrm{B}$ levels with $\mathrm{dCTApFs}$ treatment compared with untreated controls (Fig. 3I), consistent with a mechanism involving ER stress.

Next, the mechanism by which dTCApFs-induced ER stress may be associated with apoptosis was investigated by examining the ER stress repair mechanism. RT-PCR was used to determine the levels of spliced X-box-binding protein 1 (sXBP1) [a key player in the ER repair mechanism that upregulates ER-stress repair genes (9)] with dTCApFs treatment. It was observed that long-term exposure to dTCApFs led to downregulation of sXBP1 (Fig. 4A). The anti-apoptotic factor GAAP (10) was also found (by IF staining) to be downregulated with dTCApFs treatment (Fig. 4B), and cell viability (measured by the Annexin V FITC assay) was found to be reduced (Fig. 4C). In the cell viability experiments, dTCApFs was found to cause apoptosis in 50\% of PANC-1 cells. Similar results were observed in OV-90 and MDA-MB-231 cell lines.

In vivo studies: Murine xenograft models of human pancreatic cancer. In vivo studies were next performed to verify the findings of the cell culture. First, the Golgi apparatus structure was checked in tumor sections from the PANC-1 murine xenograft model, comparing dTCApFs-treated mice and controls. Similar to the cell culture findings, structural changes were observed in the ER/Golgi (Fig. 5A). Two molecular markers for ER stress [pIRE1 and $\mathrm{BiP}(6,7)]$ were also investigated; again, the in vivo results were consistent with the cell culture findings, as an increase in the number of cells expressing the markers (as detected by IHC) was observed with dTCApFs treatment (Fig. 5B). Of note, the effect of dTCApFs treatment on tumor size in the PANC-1 murine xenograft model was also examined, and it was observed that the increase in tumor size observed in control mice (no dTCApFs treatment) was attenuated in dTCApFs-treated mice (Fig. 5C).

Preliminary evidence on dTCApFs MOA from human studies. In the final set of experiments, the cell culture and in vivo findings were confirmed by analyzing human tissue samples obtained from a patient who received dTCApFs 3 times a week at increasing concentrations of 12,24 , and $48 \mathrm{mg} / \mathrm{m}^{2}$ for 11 months, as part of a recently completed phase I study. IHC analysis of tumor sections pre- and post-treatment revealed an increase in the number of cells expressing pIRE1 and BiP (Fig. 6), supporting a MOA involving ER stress.

\section{Discussion}

The present study demonstrated that dTCApFs exerts a direct effect on tumor cells by inducing apoptosis through a novel mechanism involving the Golgi apparatus. The entry of the dTCApFs molecules into the cells is mediated through the membrane receptor ST2. Inside the cells, dTCApFs molecules bind the soluble T1/ST2 receptor, which is located in the
Golgi apparatus, and induce structural changes leading to destruction of the Golgi apparatus and loss of Golgi function. Consequently, proteins accumulate in the ER, and ER stress is generated. dTCApFs also downregulates sXBP1, thus inhibiting the ER stress repair mechanism. These opposing effects of dTCApFs trigger apoptosis (an illustration of the suggested MOA is presented in Fig. 7). To the best of our knowledge, this is the first time that this unique MOA is reported for an anticancer agent. The mechanism described herein is consistent with our previous study in AML, where induction of apoptotic pathways was observed, including those mediated by caspase 8 and $\mathrm{Bcl}-2$, with TCApF treatment (1).

The entry of dTCApFs into the cells through the T1/ST2 receptor has also been demonstrated in ST2-gene knockout ovarian cancer cells (OV-90), where TCApFs treatment exerted no effect on the Golgi apparatus (unpublished data), whereas the Golgi was completely diffused with dTCApFs treatment in OV-90 control cells. Notably, the entry through the ST2 receptor also explains the selectivity of dTCApFs, as overexpression of the T1/ST2 receptor occurs in several tumor types, whereas only a weak expression of this receptor is documented in normal cells (11-13).

From a clinical perspective, the ability of dTCApFs to strongly induce ER stress supports its potential use in combination therapy, where its role would be to enhance the effect of the concurrently administered chemotherapy agents (e.g., platinum-based therapy). This approach may be useful for tumor types that are difficult to treat, such as triple-negative breast cancer. Indeed, such an approach was investigated in a phase I clinical trial evaluating the ER stress inducer nelfinavir [Viracept ${ }^{\circledR}$, Agouron Pharmaceuticals, Inc., La Jolla, CA, USA; approved by the Food and Drug Administration for the treatment of human immunodeficiency virus infection (14)], in combination with concurrent chemotherapy (cisplatinum and etoposide) plus radiation in patients with stage IIIA/IIIB non-small-cell lung cancer (15). This phase I trial demonstrated acceptable toxicity and promising activity for this regimen (15).

Notably, dTCApFs also exerts its anticancer activity through other MOAs. Preliminary unpublished data from ongoing research suggest that these mechanisms involve indirect effects through specific activation of the immune system against cancer cells. This mechanism is compatible with the known role of ST2 in the immune response.

Molecules that destroy the Golgi apparatus and activate ER-stress mediated apoptosis were previously identified. These include the antibiotics monensin (GolgiStop ${ }^{\mathrm{TM}}$ ) and brefeldin A (BFA; GolgiPlug ${ }^{\mathrm{TM}}$ ) (both from BD Biosciences, San Jose, CA, USA) (16). Monensin has been shown to destroy the Golgi apparatus by interacting with the Golgi sodium/proton exchanger, whereas BFA has been shown to cause disassembly of the Golgi complex and redistribution of secretory proteins from the cis/medial Golgi complex to the ER, thereby lePleaseading to ER stress. Both these antibiotics are very toxic and, hence, their clinical development was not pursued (17). In contrast to monensin and BFA, TCApFs was found to be safe in preclinical toxicity studies (personal communication) and was approved for human studies. Indeed, the recently completed phase I clinical trial that evaluated dTCApFs treatment in 17 patients with advanced/metastatic 
solid tumors demonstrated tolerability and suggested efficacy for dTCApFs in this setting (personal communication).

In conclusion, dTCApFs induces apoptosis of cancer cells through a novel MOA involving two opposing effects: Inducing ER stress, while downregulating the ER stress repair mechanism. These findings provide the framework for the ongoing clinical evaluation of dTCApFs as a novel anticancer therapy.

\section{Acknowledgements}

The present study was supported by ISK and Israel Chief Scientist grants (grant no. 54811). Dr J. Ohana, Dr U. Sandler, Dr G. Kass and Dr Y. Devary are employed by ISK Ltd.

\section{References}

1. Sandler U, Devary O, Braitbard O, Ohana J, Kass G, Rubinstein AM, Friedman ZY and Devary Y: NEROFE-a novel human hormone-peptide with anti-cancer activity. J Exp Ther Oncol 8: 327-339, 2010

2. Yanagisawa K, Naito Y, Kuroiwa K, Arai T, Furukawa Y, Tomizuka H, Miura Y, Kasahara T, Tetsuka T and Tominaga S: The expression of ST2 gene in helper T cells and the binding of ST2 protein to myeloma-derived RPMI8226 cells. J Biochem 121: 95-103, 1997.

3. Kumar S, Tzimas MN, Griswold DE and Young PR: Expression of ST2, an interleukin-1 receptor homologue, is induced by proinflammatory stimuli. Biochem Biophys Res Commun 235: 474-478, 1997.

4. Garlanda C, Dinarello CA and Mantovani A: The interleukin-1 family: Back to the future. Immunity 39: 1003-1018, 2013.

5. Nishitoh $\mathrm{H}$ : $\mathrm{CHOP}$ is a multifunctional transcription factor in the ER stress response. J Biochem 151: 217-219, 2012.

6. Gething MJ: Role and regulation of the ER chaperone BiP. Semin Cell Dev Biol 10: 465-472, 1999.

7. Szegezdi E, Logue SE, Gorman AM and Samali A: Mediators of endoplasmic reticulum stress-induced apoptosis. EMBO Rep 7: 880-885, 2006.
8. Novoa I, Zhang Y, Zeng H, Jungreis R, Harding HP and Ron D: Stress-induced gene expression requires programmed recovery from translational repression. EMBO J 22: 1180-1188, 2003.

9. Hirota M, Kitagaki M, Itagaki $\mathrm{H}$ and Aiba S: Quantitative measurement of spliced XBP1 mRNA as an indicator of endoplasmic reticulum stress. J Toxicol Sci 31: 149-156, 2006.

10. Carrara G, Saraiva N, Gubser C, Johnson BF and Smith GL: Six-transmembrane topology for Golgi anti-apoptotic protein (GAAP) and Bax inhibitor 1 (BI-1) provides model for the transmembrane Bax inhibitor-containing motif (TMBIM) family. J Biol Chem 287: 15896-15905, 2012.

11. Kakkar R and Lee RT: The IL-33/ST2 pathway: Therapeutic target and novel biomarker. Nat Rev Drug Discov 7: 827-840, 2008.

12. Jovanovic IP, Pejnovic NN, Radosavljevic GD, Arsenijevic NN and Lukic ML: IL-33/ST2 axis in innate and acquired immunity to tumors. Oncoimmunology 1: 229-231, 2012.

13. Bergis D, Kassis V, Ranglack A, Koeberle V, Piiper A, Kronenberger B, Zeuzem S, Waidmann $\mathrm{O}$ and Radeke HH: High serum levels of the interleukin-33 receptor soluble ST2 as a negative prognostic factor in hepatocellular carcinoma. Transl Oncol 6: 311-318, 2013.

14. Viracept (package isert): Agouron Pharmaceuticals, Inc., La Jolla, CA, 2011.

15. Rengan R, Mick R, Pryma D, Rosen MA, Lin LL, Maity AM, Evans TL, Stevenson JP, Langer CJ, Kucharczuk J, et al: A phase I trial of the HIV protease inhibitor nelfinavir with concurrent chemoradiotherapy for unresectable stage IIIA/IIIB non-small cell lung cancer: A report of toxicities and clinical response. J Thorac Oncol 7: 709-715, 2012.

16. Rosa P, Mantovani S, Rosboch R and Huttner WB: Monensin and brefeldin A differentially affect the phosphorylation and sulfation of secretory proteins. J Biol Chem 267: 12227-12232, 1992.

17. Torii S, Banno T, Watanabe T, Ikehara Y, Murakami K and Nakayama K: Cytotoxicity of brefeldin A correlates with its inhibitory effect on membrane binding of COP coat proteins. J Biol Chem 270: 11574-11580, 1995. 